International Journal of Biological Sciences

ISSN 1449-2288 www.biolsci.org 2006 2(1):23-29

Short research communication

C2006 Ivyspring International Publisher. All rights reserved

\title{
Seminal malondialdehyde concentration but not glutathione peroxidase activity is negatively correlated with seminal concentration and motility
}

\author{
Yao-Yuan Hsieh ${ }^{12}$, Chi-Chen Chang ${ }^{1}$, Chich-Sheng Lin 2
}

1. Department of Obstetrics and Gynecology, China Medical University Hospital, Taichung, Taiwan

2. Department of Biological Science and Technology, National Chiao Tung University, Hsinchu, Taiwan

Corresponding address: Chich-Sheng Lin, Ph.D. Department of Biological Science and Technology, National Chiao Tung University, 75 Po-Ai St., Hsinchu, 300 Taiwan. Tel: 886-3-5712121 ext. 56954, Fax: 886-3-5729288. E-mail: lincs@cc.nctu.edu.tw; lincs.biotech@msa.hinet.net

Received: 2006.01.09; Accepted: 2006.03.26; Published: 2006.03.27

Objectives: Reactive oxygen species (ROS) induced lipid peroxidation is associated with sperm function. Malondialdehyde (MDA) concentration and glutathione peroxidase (GPx) activity represent the lipid peroxidation and spermicidal antioxidant, respectively. We aimed to evaluate the relationship of MDA and GPx levels with sperm parameters.

Patients and methods: Specimens were divided into two groups: group 1. normospermia (n=20); group 2. oligoasthenospermia $(n=31)$. Seminal MDA concentration was measured by thiobarbituric acid reaction method. Seminal GPx activity was measured by oxidation of reduced nicotinamide-adenine dinucleotide. Seminal MDA levels and GPx activities in both groups were compared.

Results: MDA concentrations in both groups were significantly different (1.52 \pm 0.75 vs. $2.25 \pm 0.88 \mathrm{nM}, p=$ $0.0021)$. GPx activities in both groups were non-significantly different $(0.48 \pm 0.11$ vs. $0.47 \pm 0.12 \mathrm{U} / \mathrm{ml})$. MDA levels were negatively correlated with the sperm motility (MDA $=-0.014 \times$ motility $+2.62, p=0.017)$ and concentration (MDA $=-0.0045 \times$ concentration $+2.23, p=0.0166)$. GPx activities were positively but nonsignificantly correlated with the sperm concentration and sperm motility.

Conclusions: Seminal MDA concentrations are negatively correlated with sperm concentration and motility, which might provide a simple and useful tool in predicting sperm parameters. GPx activity is non-significantly correlated with the seminal quality. Roles of seminal MDA upon spermatogenesis merits further surveys.

Key words: glutathione peroxidase, malondialdehyde, oligoasthenozoospermia, semen, sperm

\section{Introduction}

Reactive oxygen species (ROS) play an important role in the human reproduction. Spermatozoa are rich in polyunsaturated fatty acids as well as susceptible to be attacked by ROS or membrane lipid peroxide ion. The equilibrium between the amounts of ROS produced and scavenged is related with the gamete cell stability and damage. Free radicals have beneficial or detrimental effects upon sperm functions, which depend on their nature and concentration. The generations of ROS, such as superoxide anion, hydrogen peroxide, and the hydroxyl radical can result in the damage to cell membranes. Spermatozoa are highly sensitive to injuries caused by high ROS concentration [1]. Excessive generation of ROS in semen may be associated with reduced sperm fertilizing potentials. Spermatozoa are rendered dysfunctional by lipid peroxidation and altered membrane function, together with impaired metabolism, morphology, and motility [2]. Excessive generation of ROS in semen, mainly by neutrophils but also by abnormal spermatozoa, could be linked with infertility.
Lipid peroxidation triggers the loss of membrane integrity, causing increased cell permeability, enzyme inactivation, structural damage to DNA, and cell death [3]. Polyunsaturated fatty acid in the phospholipids of the human spermatozooa is highly susceptible to peroxidation. Oxygen free radicals generated by spermatozoa may be involved in the production of spermicidal cytotoxic end products [4]. Human spermatozoa possess these defense enzymes, which might be useful in the prediction of sperm fertilizing potentials [5].

Numerous antioxidants are related with the ROS detoxification, including superoxide dismutase (SOD), catalase, malondialdehyde (MDA), and glutathione peroxidase (GPx). MDA, an end product of lipid peroxidation, represented the level of lipid peroxidation. High lipid peroxidation as represented by MDA levels may cause changes in the sperm and diminish fertility [5]. GPx, a selenium-containing antioxidative enzyme scavenging system, act directly as an antioxidant and an inhibitor of lipid peroxidation. GPx could protect the sperm against peroxidative damage [6]. GPx also plays an important role in sperm maturation from the early events up to 
the onset of fertilization [7]. With the absence of GPx may lead to reduce fertilizing capacity [8]. The activities of seminal GPx was lower in infertile patients than in fertile patients [9].

However, the correlation or real roles of MDA and GPx with seminal quality remains controversial [10]. Few investigators presented the role of MDA upon sperm concentration and motility. We have previously demonstrated the relationship between the SOD and sperm quality [11]. We noted that the SOD activities of sperm and seminal plasma were non-significantly correlated with the seminal quality. Herein we further tried to detect the relationship of the seminal MDA and GPx activity with spermatozoal characteristics in subfertile men. To the best of our knowledge, it is among the first few reports in this aspect.

\section{Materials and methods}

Fifty-one infertile couples who accepted the semen analyses were included. Semen was obtained by masturbation after at least 72 hours of sexual abstinence. Semen samples were collected into sterile containers for immediate transportation to the laboratory within 30 minutes after ejaculation. This series was approved by the ethical committee and institutional review board of the China Medical University Hospital. Informed consents were signed by all couples who donated their semen.

After liquefaction, samples were analyzed for volume, leukocyte count, sperm motility and morphology according to WHO guidelines [12]. Then an aliquot of $6 \mu \mathrm{l}$ of each specimen was loaded into a 30-mm microcell slide and subjected to computerassisted semen analysis (CASA) with the use of a HTM-S motility analyzer (Hamilton-Thorne Research, Beverly, MA). All semen samples were divided into 2 groups: Group 1. idiopathic infertility (normospermia, $\mathrm{n}=20$ ); Group 2. male infertility (oligo- or asthenozoospermia, $\mathrm{n}=31$, count < $20 \times 10^{6} / \mathrm{ml}$, motility $<50 \%$ ). Then the semen samples were loaded into the Eppendorf tubes $(3 \mathrm{ml})$ and stored in the liquid nitrogen tank before MDA and GPx analyses.

MDA levels were analyzed according to methods described by Rao et al. [13]. MDA was assessed using the thiobarbituric acid method. Briefly, one hundred $\mu$ l of seminal plasma was added in 0.9 $\mathrm{ml}$ of distilled water into glass tube. To each tube, 0.5 $\mathrm{ml}$ of thiobarbituric acid reagent $(0.67 \mathrm{~g}$ of $2-$ thiobarbituric acid dissolved in $100 \mathrm{ml}$ of distilled water with $0.5 \mathrm{~g} \mathrm{NaOH}$ and $100 \mathrm{ml}$ glacial acetic acid added) was added and then heated for $1 \mathrm{~h}$ in a boiling water bath. After cooling, each tube was centrifuged for $10 \mathrm{~min}$ at $4,000 \times g$ and the supernatant absorbance was read on a spectrophotometer at $534 \mathrm{~nm}$.

GPx activity was measured in accordance with our previous report [14]. In brief, the $3 \mathrm{ml}$ reaction consisted of $0.1 \mathrm{M} \mathrm{PB}(\mathrm{pH} 7.0)$ containing $1 \mathrm{mM}$
EDTA, 1.5 mM NADPH, 10 mM GSH, 0.4 U/ml GSH reductase, $12 \mathrm{mM}$ tert-butyl hydroperoxide, and 1 $\mathrm{mM} \mathrm{NaN}{ }_{3}$ to inhibit catalase activity in the sample. The tert-butyl hydroperoxide was used as substrate instead of $\mathrm{H}_{2} \mathrm{O}_{2}$. Reaction rate was determined at 340 $\mathrm{nm}$ and $37^{\circ} \mathrm{C}$ for $5 \mathrm{~min}$. Enzyme activity was expressed in unit per $\mathrm{mg}$ of protein. One unit is defined as the amount of GPx leading $1 \mu \mathrm{mol} \mathrm{GSH}$ oxidized per min.

The MDA levels and GPx activities in both groups were determined. The relationships of the MDA levels and GPx activities with the sperm motility and concentrations were also compared. The SAS system (version 8.1, SAS Institute Inc., Cary, North Carolina, USA) with $t$-test and linear regression model were utilized for statistical analyses. A $p$-value $<0.05$ was considered statistically significant.

\section{Results}

The concentrations of seminal MDA in both groups were significantly different. Individuals with oligoasthenospermia are related with the elevated MDA concentrations. The levels of MDA in Group 1 and 2 were $1.52 \pm 0.75$ and $2.25 \pm 0.88 \mathrm{nM}$, respectively (Figure $1 \mathrm{~A}, p=0.0021$ ). In contrast, the activities of seminal plasma GPx in both groups were not significantly different. The values of GPx activities in Group 1 and 2 were $0.48 \pm 0.11$ and $0.47 \pm$ $0.12 \mathrm{U} / \mathrm{ml}$, respectively (Figure $1 \mathrm{~B}, p=0.713$ ).

Figure 1. Comparisons of (A) malondialdehyde and (B) glutathione peroxidase activities in normal individuals and oligoasthenozoospermia patients.

(A)

3.5

3.0
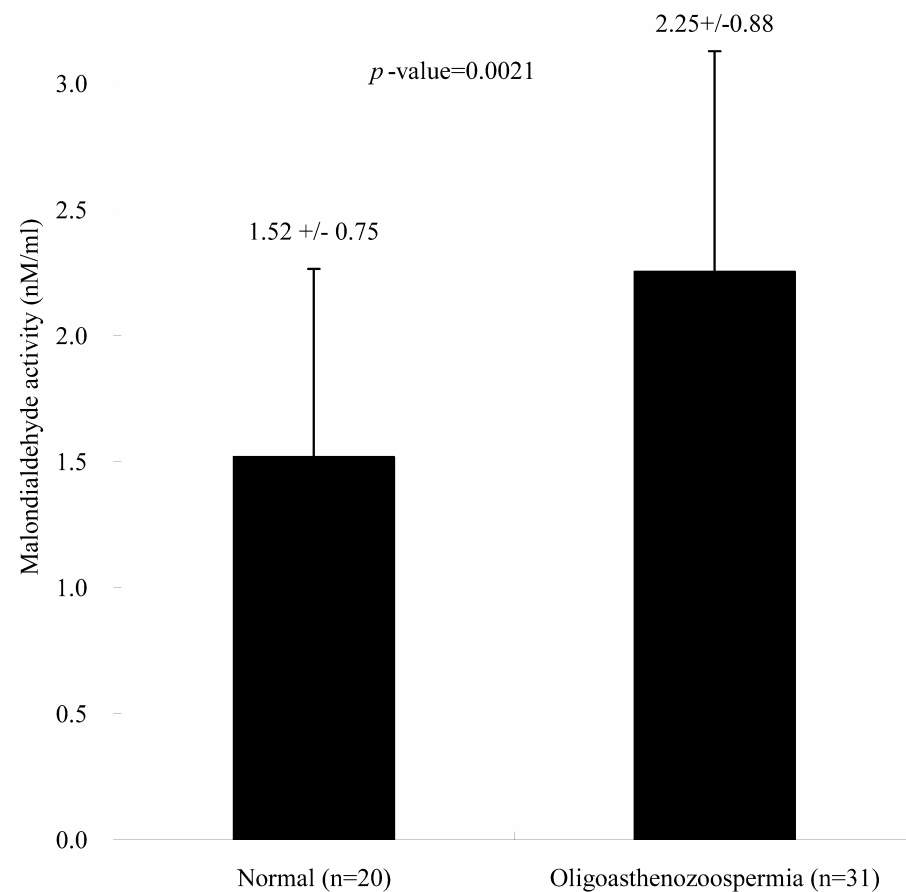
$2.25+/-0.88$ 


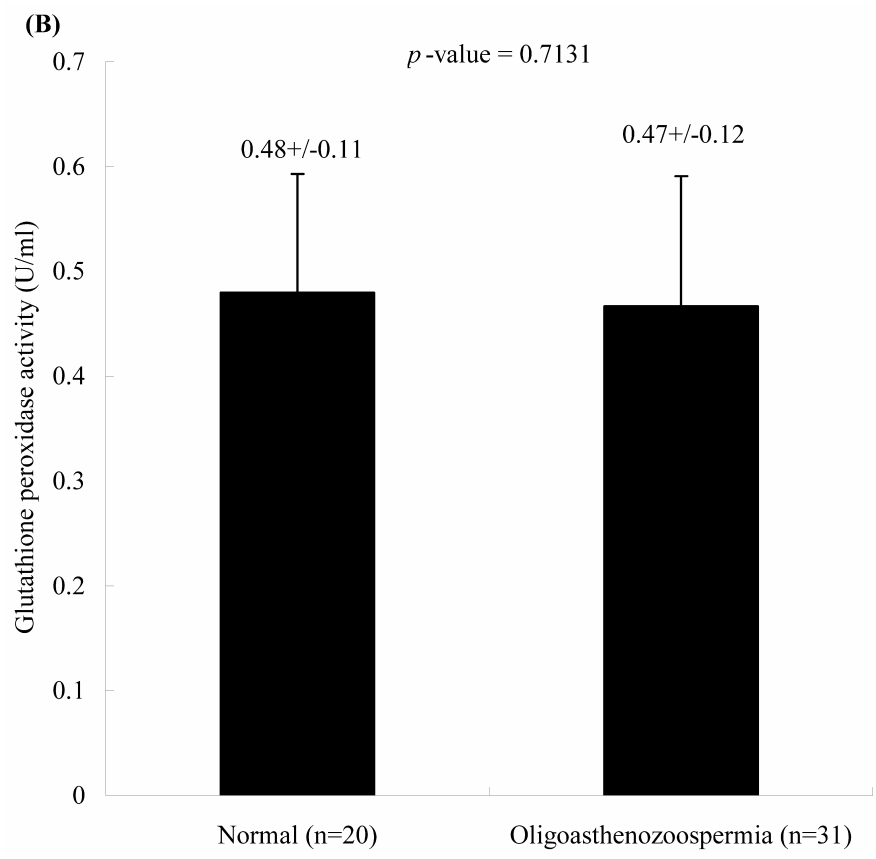

Sperm concentration is negatively correlated with MDA level. The formula of their relationship was: MDA $=-0.0045 \times$ sperm concentration +2.23 (Figure $2 \mathrm{~A}, p=0.0166$ ). Sperm concentration is positively but nonstatistically related with GPx level. The formula of their relationship was: GPx $=0.0009 \times$ sperm concentration + 0.42 (Figure 2B, $p=0.0696$ ). We noted the negative correlation between the MDA activity and sperm motility. We establish the formula of their relationship: MDA $=-0.014 \times$ sperm motility +2.62 (Figure $3 \mathrm{~A}, p=0.017$ ). We also noted the positive, but non-significant correlation between the sperm motility and the GPx activity. The formula of their relationship was: GPx $=0.0012 x$ sperm motility +0.42 (Figure $3 \mathrm{~B}, p=0.134$ ).

Figure 2. The correlations of the sperm concentration with (A) malondialdehyde and (B) glutathione peroxidase activities.
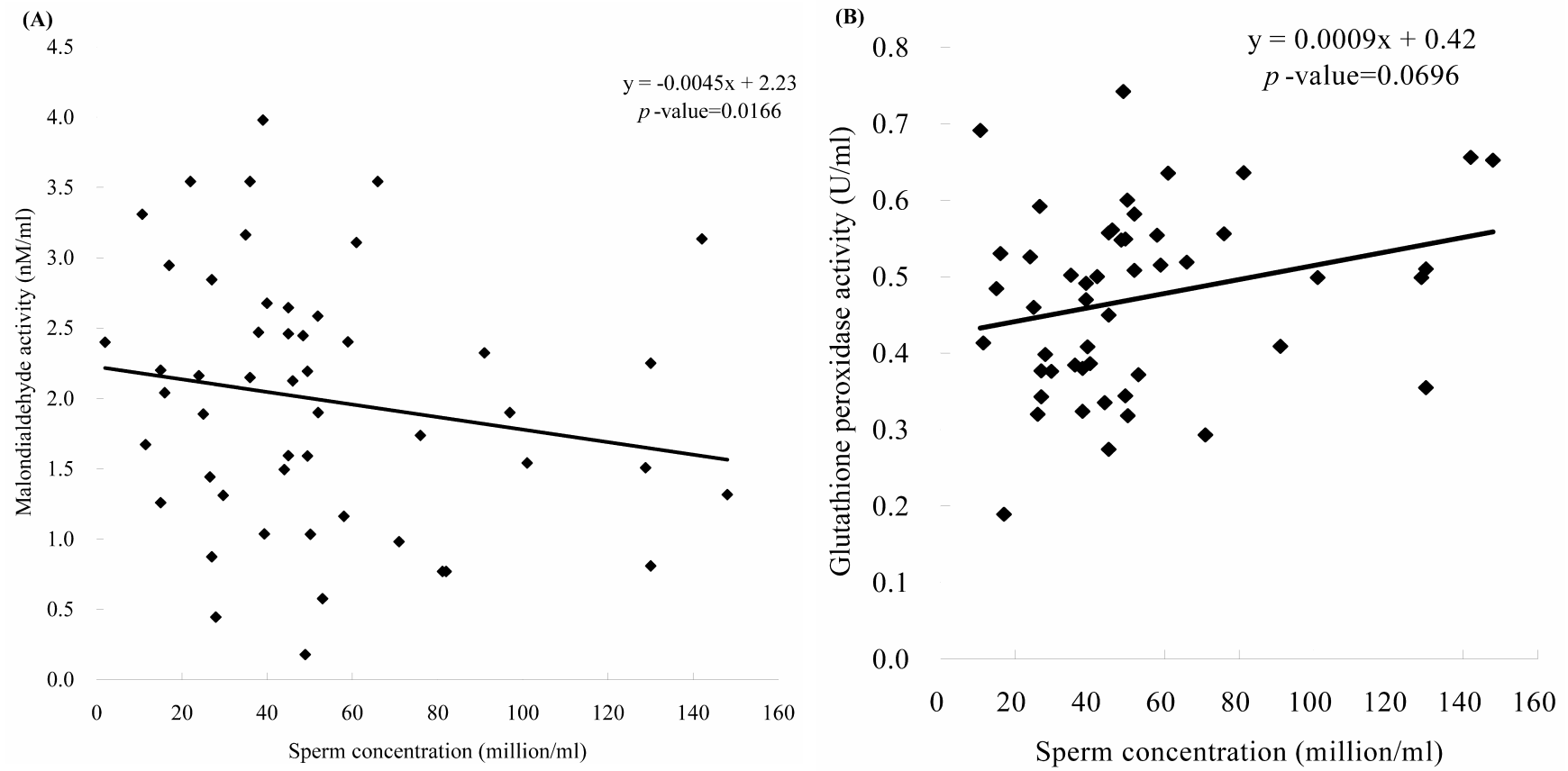
Figure 3. The correlations of the sperm motility with (A) malondialdehyde and (B) glutathione peroxidase activities
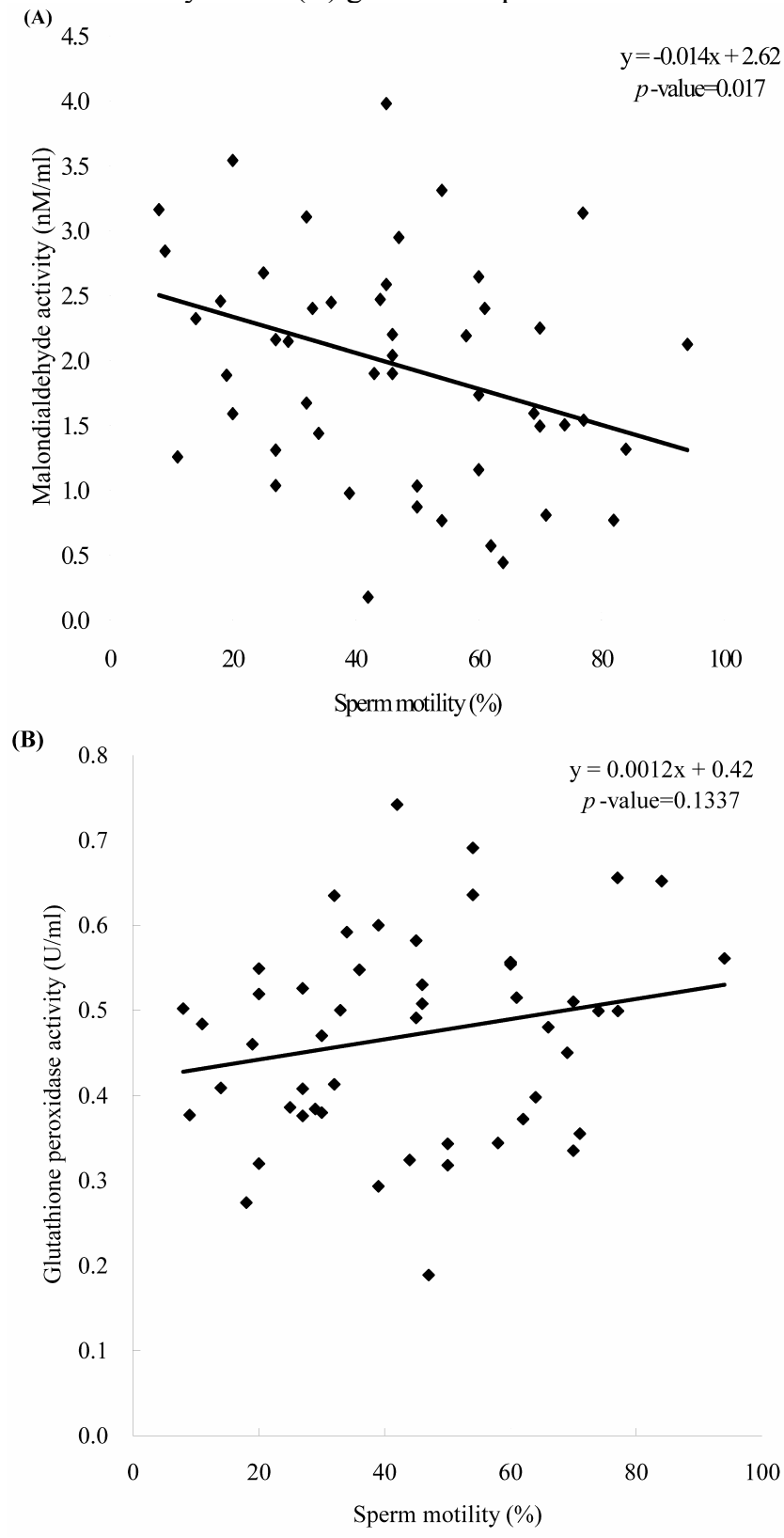

\section{Discussion}

Oxidative damage is common for spermatozoa during epididymal maturation and storage. Human spermatozoa are highly susceptible to oxidative injury but are naturally protected from such injury by the antioxidant properties of seminal plasma. ROS plays a central for sperm physiology such as sperm maturation and capacitation. Abnormal ROS production is associated with defective sperm function [15, 16]. A fine balance between ROS production and recycling is essential for spermatogenesis. Excessive generation of seminal ROS, mainly by neutrophils but also by immobile sperm, morphologically abnormal sperm, or morphologically normal but functionally abnormal sperm, could be a cause for male infertility [17]. The incidence of spontaneous pregnancy was negatively correlates with ROS production [18]. In fact, ROS have beneficial or detrimental effects on sperm functions depending on the nature and concentration of the ROS involved, as well as the moment and the location of exposure [19].

Antioxidants in semen serve to protect ejaculated spermatozoa from oxidative stress such as that which occurs in the female reproductive tract. The lipid peroxidation of unsaturated fatty acids in sperm membranes is one of the most important effects from ROS-induced cell damage what may lead to persistent infertility [20]. Lipid peroxidation was increased during in-vitro storage of spermatozoa, which might impair sperm motility [21]. Camejo et al. [22] observed a significant positive correlation between seminal IL-6 levels and lipid peroxidation of sperm membrane. The high level cytokines produced by human sperm and leucocytes might further stimulate the ROS production. Exposure of spermatozoa to ROS has been associated with cellular injury, which includes DNA damage and lipid peroxidation [23]. ROS may affect the integrity of the sperm genome and induce high frequencies of single and double DNA strand breaks [24].

ROS is known to play a major role in the etiology of defective sperm function via mechanisms involving the induction of peroxidative damage to the plasma membrane [25]. ROS is related with the spermatozoa hyperactivation, capacitation and acrosome reaction [26]. ROS production is negatively related with the sperm-oocyte fusion capacity of human spermatozoa [27]. ROS at low concentrations may inhibit sperm-egg fusion via oxidation of the $\mathrm{SH}$ proteins in the sperm membrane [28]. High lipid peroxidation may reduce the capacity of the sperm to undergo acrosomal reaction and to fertilize [27]. The spermatozoa capability for oocyte fusion was impaired with the addition of hydrogen peroxide [29].

Antioxidant enzymes, such as SOD and GPx, could dispose hydroperoxide and other ROS. A balance is maintained between the amount of ROS and that scavenged by antioxidant. Cellular damage arises when this equilibrium is disturbed, especially when the cellular scavenging systems cannot eliminate the increased ROS. Lipid peroxidation could damage the cell plasma membrane, which leads to loss of cytosolic components and cell death. Human spermatozoa possess the antilipoperoxidative enzymes, including SOD and GPx. These enzymes could dispose hydroperoxide and other ROS. Normally, a balance is maintained between the amount of ROS and that scavenged by antioxidant. Cellular damage arises when this equilibrium is disturbed, especially when the cellular scavenging systems cannot eliminate the increased ROS.

Among the various methods for detection of lipid peroxidation, we chose to measure spontaneous MDA production, which reflects the peroxidation of 
polyunsaturated phospholipids, the major components of sperm membrane [2]. MDA measurements are physiological and relevant because major loss of sperm function may occur with minimal damage to the membranes that envelop the sperm and/or divide key intracellular sperm compartments. Geva et al. [5] demonstrated that the high MDA production in the male with low fertilization rates in their previous IVF cycles. They also found that the reduction of MDA by using antioxidant therapy was correlated with the improvement of fertilization rates.

There is controversy about the seminal MDA activity and the sperm quality. Kobayashi et al. [32] demonstrated that MDA concentration in spermatozoa was significantly related to the number of immotile spermatozoa. Elevated seminal MDA concentration was noted in patients with oligoasthenoteratozoospermia [20]. In contrast, Suleiman et al. [33] demonstrated that the MDA concentration in the seminal plasma was not correlated with the sperm concentration and motility. In this series, we found the different concentrations of MDA between the samples of normo- and oligoasthenozoospermoa. We observed the negative correlation between the MDA activity and sperm motility or concentration, which was compatible with the findings of Geva et al. [5], Fraczek et al. [20], and Kobayashi et al. [32].

Oligoasthenozoosperm is associated with higher seminal MDA activity. It suggested that the lipid peroxidation, which was represented by the MDA activity, might compromise the sperm viability. Increased MDA activity could represent the pathologic lipid peroxidation of spermatozoa membrane and the following inhibition of sperm motility and viability. Some agents used for improving the sperm qualities could influence the MDA activity. Administration of vitamin E may decrease the MDA activity with concomitant increased sperm motility and viability [30]. Administration of pentoxifylline could decrease the generation of ROS, lipid peroxidation and MDA in human spermatozoa [31]. These suggested that the vitamin $\mathrm{E}$ and pentoxifylline might improve the motility and fertilization rate of sperm by reducing the lipid peroxidation [5].

GPx , a ROS scavenger, plays a role in protecting sperm membranes from the deleterious effects of lipid peroxidation [34]. GPx is related with the balance between the GSSG (reduced form of glutathione) and GSH (oxidized form of glutathione) [10]. Glutathione acts as a free radical scavenger and improves the semen quality. Glutathione could inhibit the lipid peroxidation of the cell membrane [10]. Reduced glutathione could protect spermatozoa from oxidative stress [35]. Through the interaction with GPx, reduced glutathione can neutralize hydroxyl radicals and detoxify the peroxides [10].

In subfertile patients, increased leukocytes in semen were associated with lower GPx activity [36]. GPx activities were significantly elevated when the semen samples contained $<10^{6}$ leukocytes $/ \mathrm{ml}$ [36]. It has been suggested that higher ROS production in infertile patients may be due to increased ROS production rather than defective ROS scavenging activity [37]. GPx activity in follicular fluid from infertile women was significantly lower than in fertile women [38]. GPx is also associated with the nuclear function of sperm [39]. GPx could scavenges lipid peroxides released form the plasma membrane and further protect the DNA from oxidative attack [40]. Suppressed GPx activity may allow the production of the peroxides from the sperm plasma membrane to the chromatin where they could induce DNA strand breakage [40].

Recently, Dandekar et al. [41] demonstrated that the GPx concentration correlate positively with asthenozoospermia. Higher GPx may catalyze the ROS, which might improve the sperm motility. Increased spermatozoa might produce higher level of GPx. These could interpret the positive but nonsignificant correlation between GPx and sperm motility and concentration in this study. Giannattasio et al. [42] demonstrate that the seminal GPx activity from healthy subjects was 10 times greater than that from infertile males. In contrast, Tramer et al., [43] demonstrated the non-difference of GPx activities between normo- and hypomotile human sperm samples. In this study, we could not observe their statistical correlations. It suggests that the seminal GPx is still not a useful tool in determining sperm fertilizing potential. This may be due to the case limitation or the complex interactions between the ROS and numerous antioxidants. In fact, the regulation of sperm function and the mechanisms involved may be complex and multifactorial.

In conclusion, the MDA level representing lipid peroxidation is negatively correlated with the sperm motility and concentration. In contrast, the GPx activities are not significantly correlated with the sperm motility or concentration. This could provide the database about the effects of MDA and GPx upon sperm. Increased activity of seminal MDA may be correlated or even responsible for the pathophysiology of male infertility. Furthermore, seminal MDA survey is a simple and useful screening tool in determining sperm fertilizing potentials. However, the regulation of sperm function and the mechanisms involved may be complex and multifactorial. Real role of MDA, GPx and other antioxidants upon the sperm qualities merits further surveys.

\section{Conflict of interest}

The authors have declared that no conflict of interest exists.

\section{References}

1. Aiken RJ, Clarkson JS. Cellular basis of defective sperm function and its association with the genesis of reactive oxygen species by human spermatozoa. J Reprod Fertil 1987;81:459-69. 
2. Cummins JM, Jequier AM, Kan R. Molecular biology of human male infertility: links with aging, mitochondrial genetics and oxidative stress. Mol Reprod Dev 1994;37:345-62.

3. Halliwell B. Free radicals, antioxidants, and human disease: curiosity, cause, or consequence? Lancet 1994;344:721-4.

4. Selly MU, Lacey MJ, Bartlett MR, Copeland CM, Ardlie NG. Content of significant amounts of a cytotoxic end-product of lipid peroxidation in human semen. J reprod Fertil 1991;92:291-8.

5. Geva E, Lessing JB, Bartoov B, Lerner-Geva L, Zabludovsky $\mathrm{N}$, Amit A. The effect of antioxidant treatment on human spermatozoa fertilization rate in an in vitro fertilization program. Fertil Steril 1996;66:430-4.

6. de Lamirande E, Gagnon C. Human sperm hyperactivation in whole semen and its association with low superoxide scavenging capacity in seminal plasma. Fertil Steril 1993, 59:1291-5.

7. Vernet P, Faure J, Dufaure JP, Drevet JR. Tissue and developmental distribution, dependence upon testicular factors and attachment to spermatozoa of GPX5, a murine epididymis-specific glutathione peroxidase. Mol Reprod Dev 1997;47:87-98.

8. Hall L, Williams K, Perry AC, Frayne J, Jury JA. The majority of human glutathione peroxidase type 5 (GPX5) transcripts are incorrectly spliced: implications for the role of GPX5 in the male reproductive tract. Biochem J 1998;333:5-9.

9. Alkan I, Simsek F, Haklar G, Kervancioglu E, Ozveri H, Yalcin $\mathrm{S}$, Akdas A. Reactive oxygen species production by the spermatozoa of patients with idiopathic infertility: relationship to seminal plasma antioxidants. J Urol 1997;157:140-3.

10. Sharma RK, Agarwal A. Role of reactive oxygen species in male infertility. Urology 1996;48:835-50.

11. Hsieh YY, Sun YL, Chang CC, Lee YS, Tsai HD, Lin CS. Superoxide dismutase activities of spermatozoa and seminal plasma are not correlated with male infertility. J Clin Lab Anal 2002;16:127-31.

12. World Health Organization. World Health Organization Laboratory Manual for the Examination of Human Semen and Semen-Cervical Mucus Interaction, 3rd ed. Cambridge: Cambridge University Press, 1992.

13. Rao B, Souflir JC, Martin M, David G. Lipid peroxidation in human spermatozoa as related to midpiece abnormalities and motility. Gamete Res 1989;24:127-34.

14. Lin CS, Liu CY, Sun YL, Chang LC, Chiu YT, Huang SY, Lin JH, Yang PC, Chu R, Huang MC and Mao SJT. Alteration of endogenous antioxidant enzymes in naturally occurring hypertrophic cardiomyopathy. Biochem Mo Biol Int 1997;43:1253-63.

15. Iwasaki A, Gagnon C. Formation of reactive oxygen species in spermatozoa of infertile patients. Fertil Steril 1992;57:409-16.

16. Mazzilli F, Rossi T, Marchesini M, Ronconi C, Dondero F. Superoxide anion in human semen related to seminal parameters and clinical aspects. Fertil Steril 1994;62:862-8.

17. Plante M, de Lamirande E, Gagnon C. Reactive oxygen species released by activated neutrophils, but not by deficient spermatozoa, are sufficient to affect normal sperm motility. Fertil Steril 1994;62:387-93.

18. Aitken RJ, Irvine DS, Wu FC. Prospective analysis of spermoocyte fusion and reactive oxygen species generation as criteria for the diagnosis of infertility. Am J Obstet Gynecol. 1991;164:542-51.

19. de Lamirande E, Gagnon C. Impact of reactive oxygen species on spermatozoa: a balancing act between beneficial and detrimental effects. Hum Reprod 1995;10 (Suppl 1):15-21.

20. Fraczek M, Szkutnik D, Sanocka D, Kurpisz M. Peroxidation components of sperm lipid membranes in male infertility. Ginekol Pol 2001;72:73-9.

21. Douard V, Hermier D, Magistrini M, Blesbois E. Reproductive period affects lipid composition and quality of fresh and stored spermatozoa in Turkeys. Theriogenology 2003;59:75364.

22. Camejo MI, Segnini A, Proverbio F. Interleukin-6 (IL-6) in seminal plasma of infertile men, and lipid peroxidation of their sperm. Arch Androl 2001;47:97-101.

23. Potts RJ, Notarianni LJ, Jefferies TM. Seminal plasma reduces exogenous oxidative damage to human sperm, determined by the measurement of DNA strand breaks and lipid peroxidation. Mutat Res 2000;447:249-56.

24. Twigg JP, Irvine DS, Aitken RJ. Oxidative damage to DNA in human spermatozoa does not preclude pronucleus formation at intracytoplasmic sperm injection. Hum Reprod. 1998;13:1864-71.

25. Griveau JF, Le Lannou D. Reactive oxygen species and human spermatozoa: physiology and pathology. Int J Androl 1997;20:61-9.

26. Griveau JF, Renard P, Le Lannou D. Superoxide anion production by human spermatozoa as a part of the ionophoreinduced acrosome reaction process. Int J Androl. 1995;18:6774.

27. Aitken RJ, Clarkson JS, Fishel S. Generation of reactive oxygen species, lipid peroxidation, and human sperm function. Biol Reprod. 1989;41:183-97.

28. Mammoto A, Masumoto N, Tahara M, Ikebuchi Y, Ohmichi M, Tasaka K, Miyake A. Reactive oxygen species block spermegg fusion via oxidation of sperm sulfhydryl proteins in mice. Biol Reprod 1996;55:1063-8

29. Aitken RJ, Buckingham D, Harkiss D. Use of a xanthine oxidase free radical generating system to investigate the cytotoxic effects of reactive oxygen species on human spermatozoa. J Reprod Fertil 1993;97:441-50

30. Verma A, Kanwar KC. Effect of vitamin $E$ on human sperm motility and lipid peroxidation in vitro. Asian J Androl 1999;1:151-4

31. McKinney KA, Lewis SE, Thompson W. The effects of pentoxifylline on the generation of reactive oxygen species and lipid peroxidation in human spermatozoa. Andrologia 1996;28:15-20

32. Kobayashi T, Miyazaki T, Natori M, Nozawa S. Protective role of superoxide dismutase in human sperm motility: superoxide dismutase activity and lipid peroxide in human seminal plasma and spermatozoa. Hum Reprod 1991;6:987-91 
33. Suleiman SA, Ali ME, Zaki ZM, el-Malik EM, Nasr MA. Lipid peroxidation and human sperm motility: protective role of vitamin E. J Androl 1996;17:530-7

34. Alvarez JG, Storey BT. Role of glutathione peroxidase in protecting mammalian spermatozoa from loss of motility caused by spontaneous lipid peroxidation. Gamete Res. 1989;23:77-90.

35. Baker HW, Brindle J, Irvine DS, Aitken RJ. Protective effect of antioxidants on the impairment of sperm motility by activated polymorphonuclear leukocytes. Fertil Steril 1996;65:411-9.

36. Therond P, Auger J, Legrand A, Jouannet P. alpha-Tocopherol in human spermatozoa and seminal plasma: relationships with motility, antioxidant enzymes and leukocytes. Mol Hum Reprod 1996;2:739-44

37. Zini A, de Lamirande E, Gagnon C. Reactive oxygen species in semen of infertile patients: levels of superoxide dismutaseand catalase-like activities in seminal plasma and spermatozoa. Int J Androl. 1993;16:183-8.

38. Paszkowski T, Traub AI, Robinson SY, McMaster D. Selenium dependent glutathione peroxidase activity in human follicular fluid. Clin Chim Acta 1995;236:173-80.

39. Godeas C, Tramer F, Micali F, et al. Phospholipid hydroperoxide glutathione peroxidase (PHGPx) in rat testis nuclei is bound to chromatin. Biochem Mol Meed 1996;59:11824.

40. Twigg J, Fulton N, Gomez E, Irvine DS, Aitken RJ. Analysis o the impact of intracellular reactive oxygen species generation on the structural and functional integrity of human spermatozoa: lipid peroxidation, DNA fragmentation and effectiveness of antioxidants. Hum Reprod 1998;13:1429-36.

41. Dandekar SP, Nadkarni GD, Kulkarni VS, Punekar S. Lipid peroxidation and antioxidant enzymes in male infertility. J Postgrad Med. 2002;48:186-90

42. Giannattasio A, De Rosa M, Smeraglia R, Zarrilli S, Cimmino A, Di Rosario B, Ruggiero R, Colao A, Lombardi G. Glutathione peroxidase (GPX) activity in seminal plasma of healthy and infertile males. J Endocrinol Invest. 2002 Dec;25(11):983-6.

43. Tramer F, Caponecchia L, Sgro P, Martinelli M, Sandri G, Panfili E, Lenzi A, Gandini L. Native specific activity of glutathione peroxidase (GPx-1), phospholipid hydroperoxide glutathione peroxidase (PHGPx) and glutathione reductase (GR) does not differ between normo- and hypomotile human sperm samples. Int J Androl. 2004;27:88-93. 